\title{
The Control Method on Current Rate of Change under Constant Power
}

\section{Load in Aviation Power Grid}

\author{
Jian Zhang ${ }^{1, a^{*}, \text { Yuanjun Zhou }}{ }^{2, b}$ \\ ${ }^{1}$ College of Automation, Beihang University, Beijing, China \\ ${ }^{2}$ College of Automation, Beihang University, Beijing, China \\ a849829950@qq.coml, bzhouyuanjun@buaa.edu.cn
}

Keywords: Constant power load, The control method on current rate of change, Stability

Abstract. Closed-loop controlled electrical drive systems require constant speed without interference, which corresponds to CPLs and performs negative impedance characteristics, so that they have great influence on the stability of aviation power grid. The main purpose of this paper is to solve this problem by adopting the control method on current rate of change which makes it possible to realize the requirements of stability on aviation power grid. In addition, the paper proved the correct and efficiency of this method by using simulation.

\section{Introduction}

Constant power load (CPL) is one of the typical loads of more electric aircraft. Closed-loop controlled electrical drive systems require constant speed without interference, therefore, they correspond to CPLs, which perform negative impedance characteristics and have great influence on the stability of aviation power grid. In addition, electric driving system adopt pulse-width modulation (PWM), so there must be EMI (electromagnetic interference) filters used on the bus side, which would have a great impact on air grid stability.

In recent years, lots of papers put forward solutions on the stability problems caused by CPLs. One is to increase filter capacitor, which has been widely studied due to its sample realization [1]. According to Brayton - Moser mixed potential theory, under the condition of constant filter resonant frequency, loads can satisfy the impedance stability criterion by deploying larger capacitance to achieve the stability of network. However, larger capacitance may cause a great impact in the grid voltage and current when there is a disturbance and lead to a bad performance of the power grid. Besides, electrolytic capacitors are commonly used as large capacitor, which is difficult to ensure the high reliability requirement. To sum up, increasing the filter capacitance cannot fully meet the GJB's requirements for grid transient shock and reliability.

Negative impedance compensation method is also proposed as a new method [2]. This method transfers the negative impedance into positive impedance through sampling the voltage signal behind the filter, which is connected to the closed loop control system by the compensator to compensate current. The grid voltage dynamic performance is improved greatly in this way, but as the amount of compensation works on the current loop, when there is a disturbance, grid current transient would exceed the scope of requirements and affect the quality of power grid.

In order to fully meet the requirements of grid stability in aircraft power supply system, this paper proposes a new solution, namely, adding current rate of change control loop with a limiting link to the closed-loop system. The negative impedance value can be directly controlled by controlling the current rate of change, so as to solve the power grid stability problems caused by the 
negative impedance characteristics. The analysis on admittance model of the system without current rate of change loop and the influence of the LC filter parameters on power grid transient are carried on for comparison. Firstly, the principle of current rate of change control method is analyzed in this paper. Then a simulation model is established to verify this control scheme. Finally, the conclusion is drawn.

\section{The Control Method on Current Rate of Change}

CPL's dynamic resistance $R$ can be represented through the differential of the input voltage to the input current, which performs negative resistance characteristics and is shown in Eq. 1.

$$
R=\frac{\Delta v_{L}}{\Delta i_{L}}=-\frac{v_{L}^{2}}{P} \leq 0
$$

According to Eq. 1, by controlling current rate of change $\Delta i_{L}, R$ can be adjusted to a appropriate range, and the objective of improving the stability of the power grid can be achieved. The control method on current rate of change adds an extra current rate of change loop on the basis of the original closed-loop system, meaning to control the changing rate of current of CPLs.

When mutations occur to the grid, grid voltage and current are disturbed. The disturbance is often characterized by severe shocks, namely, the current rate of change $\Delta i_{L}$ is very large, and $/ R /$ is small, so that the dynamic process is unsatisfied for some time after the disturbances, or even instability is caused by a very small $/ R /$. By acceding the rate of change of current control loop, $\Delta i_{L}$ is controlled, and the value of $/ R /$ can be increased to improve the dynamic characteristics as much as possible under the precondition of satisfy the system stability.

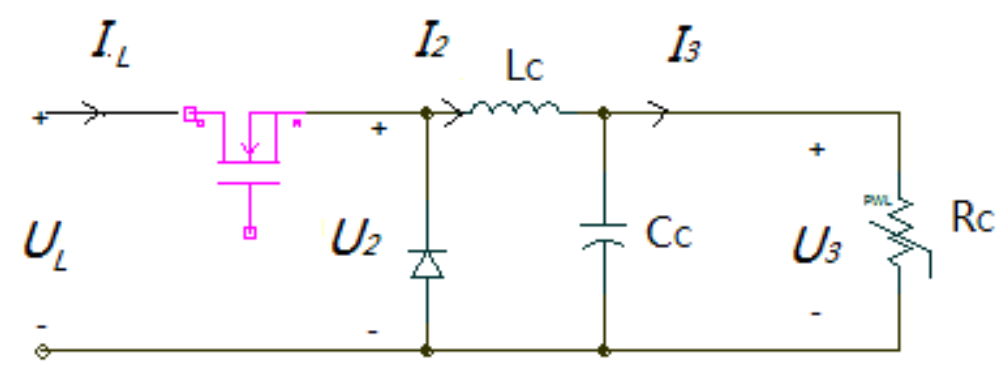

Fig.1 Chopper-type CPL circuit

Fig. 1 shows a Chopper-type CPL circuit, Eq. 2 could be drawn.

$$
\frac{U_{3}}{U_{2}}=\frac{1}{1+s \frac{L_{c}}{R_{c}}+s^{2} L_{c} C_{c}}
$$

And $U_{2}$ and $U_{L}$ satisfys Eq. 3 .

$$
U_{2}=K_{d} U_{L}
$$


$K_{d}$ is duty ratio. Due to the high frequency of PWM signal, $K_{d}$ is constant within the scope of the control band of the closed-loop control system. By small-signal linearization of Eq. 3, Eq. 4 is obtained.

$$
\Delta U_{2}=\frac{\partial U_{2}}{\partial K_{d}} \Delta K_{d}+\frac{\partial U_{2}}{\partial U_{L}} \Delta U_{L}=U_{L 0} \Delta K_{d}+K_{d 0} \Delta U_{L}
$$

Subscripts ${ }^{0}$ represents the steady state value of variables. The current relationship can be got in the same way, which is shown in Eq. 5.

$$
\Delta I_{L}=I_{20} \Delta K_{d}+K_{d 0} \Delta I_{2}
$$

Because transient process is mainly discussed in this paper, and the duration is very short, so integral elements are ignored, we consider the current regulator and the current rate regulator both are proportion links, namely $G_{1}=k_{1}$ and $G_{2}=k_{2}$. The system model of current rate of change control method is as shown in Fig. 2, and the admittance model is shown in Eq. 6.

$$
Y_{i n}=\frac{1}{R}=\frac{\Delta I_{L}}{\Delta U_{L}}=K_{d 0} \frac{\frac{K_{d 0}}{L}\left(1-\frac{k_{2} I_{20}}{R_{c} C_{c} K_{d 0}}\right) s+\frac{K_{d 0}-k_{1} k_{2} I_{20}}{R_{c} L_{c} C_{c}}}{s^{2}+\frac{1}{R_{c} C_{c}}\left(1+\frac{U_{10} k_{2}}{L_{c}}\right) s+\frac{1}{L_{c} C_{c}}+\frac{U_{10} k_{1} k_{2}}{R_{c} L_{c} C_{c}}}
$$

Damping Factor of $Y_{i n}$ is shown in Eq. 7.

$$
\xi=\frac{1}{2} \sqrt{\frac{L_{c}}{C_{c}} * \frac{1}{R_{c}} * \frac{1}{\left(R_{c}+U_{10} k_{1} k_{2}\right)}}
$$

According to Eq. 7, $\xi$ increases when $\left|k_{2}\right| \leq 1$, which has good effect on the characters of the dynamic process.

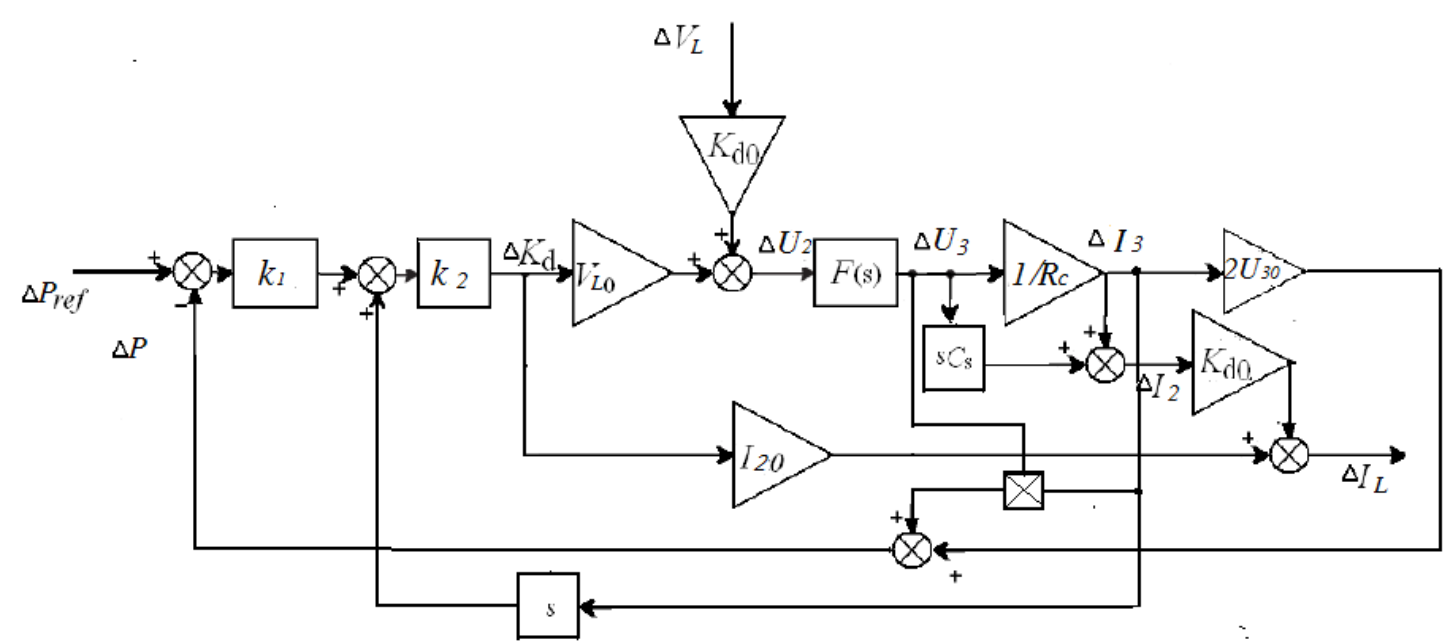

Fig.2 The system model of current rate of change control method 
The step response curves are drawn with different value of $k_{1}$ and $k_{2}$, which is shown in Fig.3. The steady-state CPL's equivalent admittances are the same in these three conditions, but CPL's equivalent conductance decreases and the adverse effects on the grid reduce in the dynamic process through adding the rate of change of current control loop and selecting a reasonable amplification coefficient.

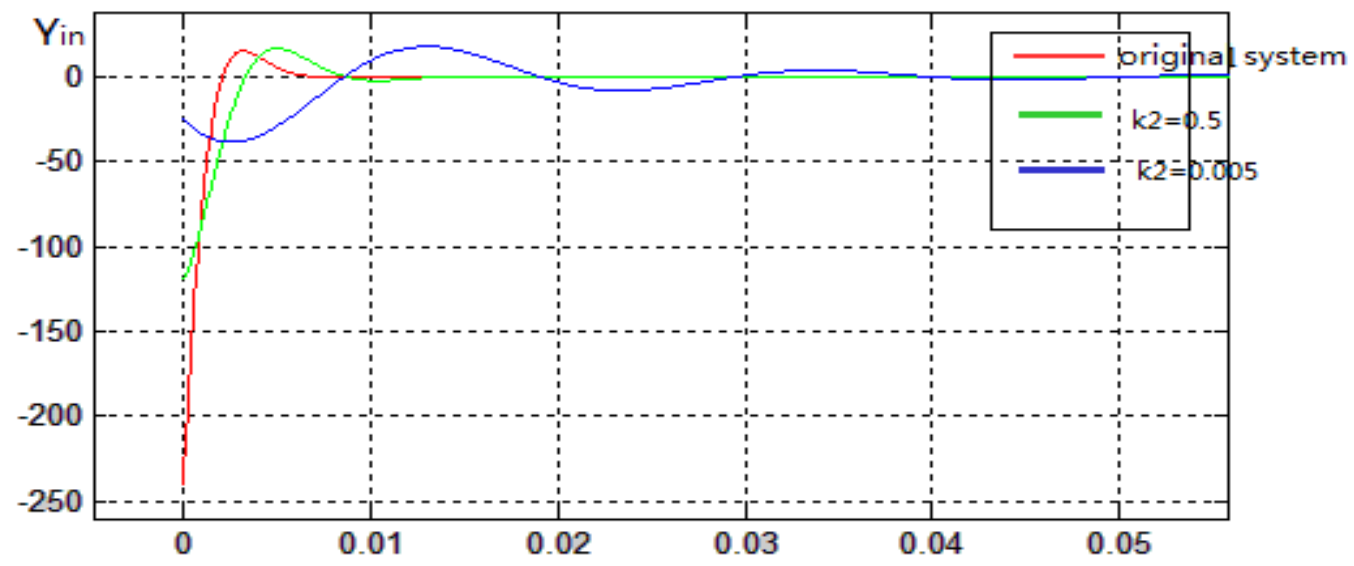

Fig.3 the step response curves

\section{Simulation Results}

Build models according to Fig. 2 in Saber software to verify the current control method proposed in this paper. Power system transient index is, transient voltage amplitude $\leq 20 \mathrm{~V}$, and transient current amplitude $\leq 60 \mathrm{~A}$.

$10 \mathrm{KW}$ Chopper-type CPL is controlled by closed loop. The load stable voltage is $135 \mathrm{~V}$. The CPL is put into the 270V power grid at 0.1s. Simulation results are shown in Fig. 4 and Fig. 5.

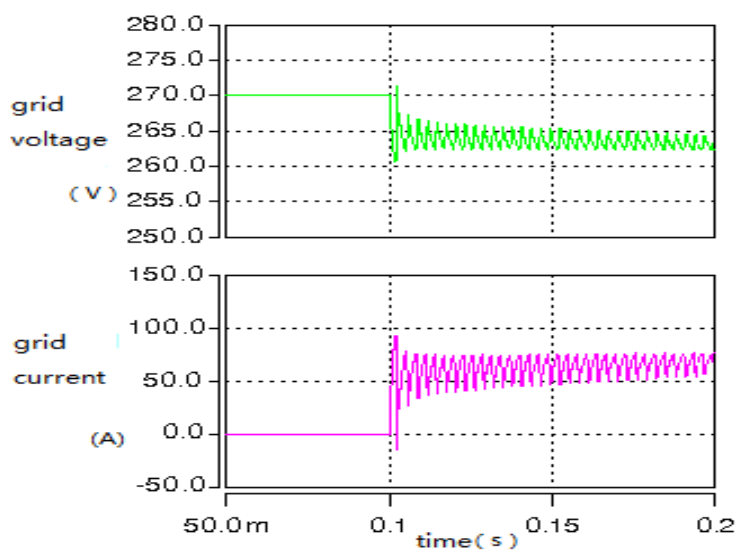

(1) original system

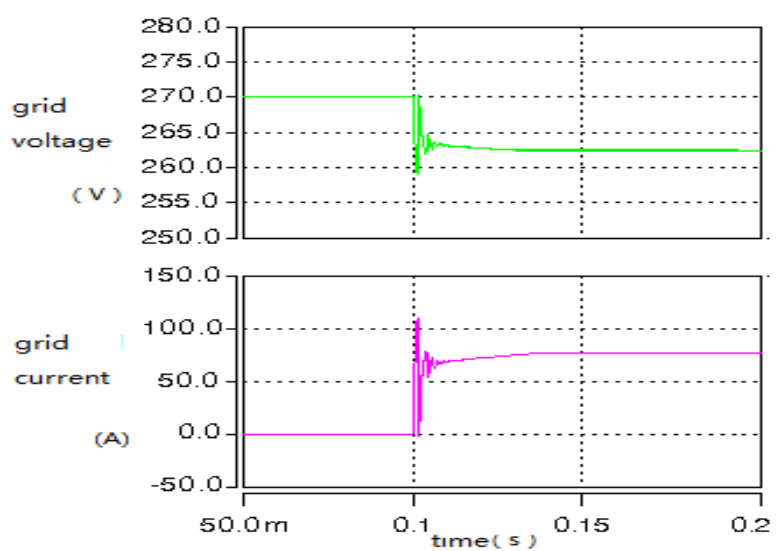

(2) the system with a larger capacitor

Fig. 4 the grid voltage and current waveform 


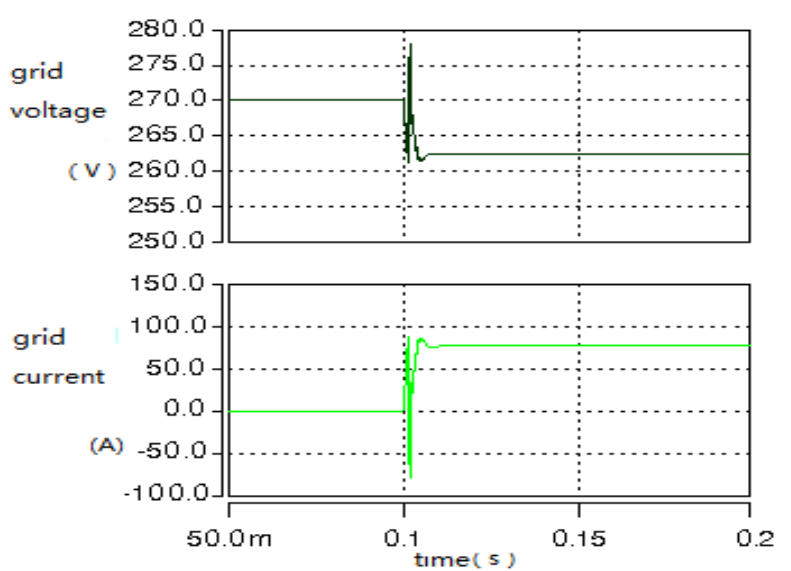

(1) $k_{2}=2$

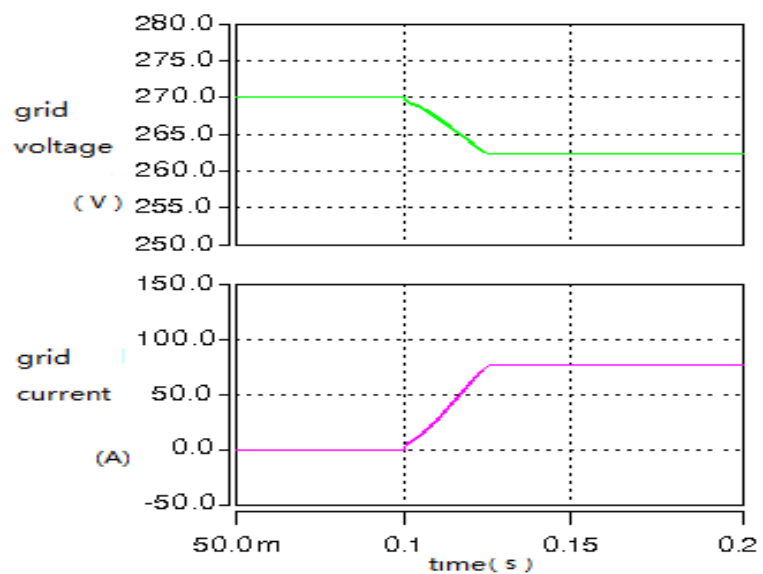

(3) $k_{2}=0.05$

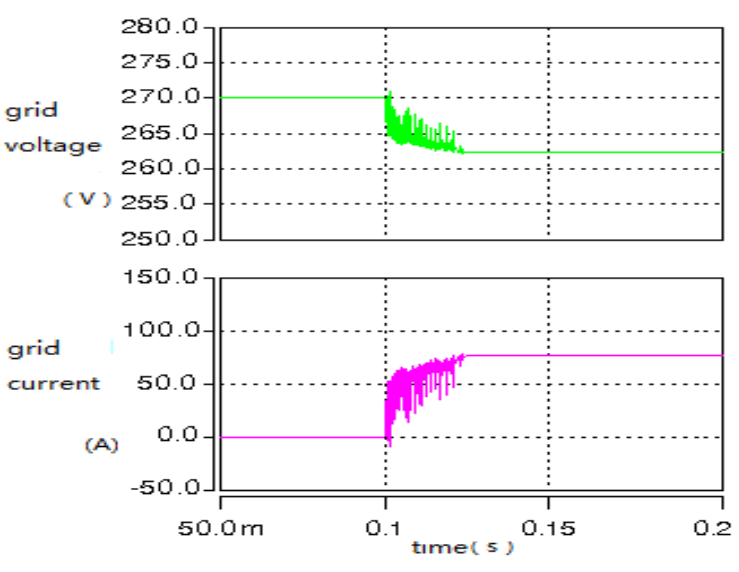

(2) $k_{2}=0.5$
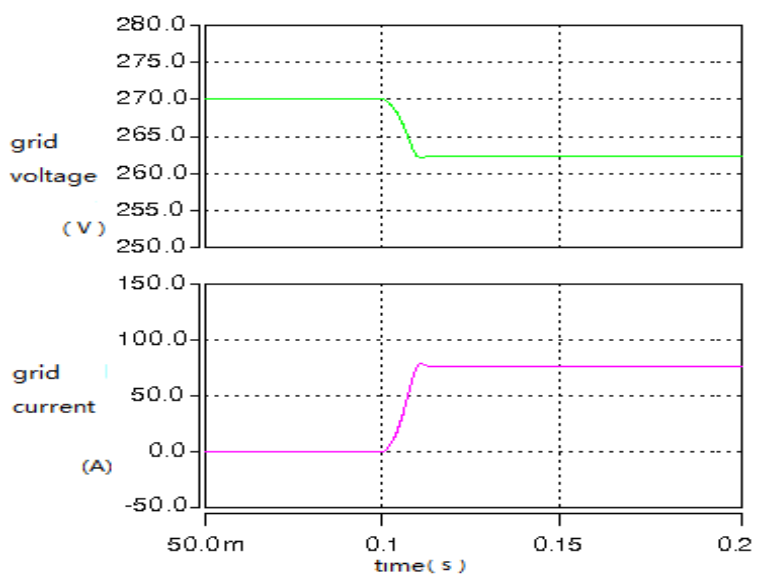

(4) the improved control method $\left(k_{2}=0.5\right)$

Fig. 5 the grid voltage and current waveform

Fig. 4 (1) shows the grid voltage and current waveform of the original current closed loop system when CPL is suddenly put into the grid. Negative impedance characteristics of CPL cause an oscillation of the power grid voltage and current, especially the current turmoil is beyond the power grid transient index, which makes the dynamic performance unsatisfied. Fig. 4 (2) shows the simulation waveform under the condition of increasing the filtering capacity and reducing the filter inductance. It can be seen that increasing the filtering capacity significantly reduce the oscillation of the transient response time and improve the grid voltage and current characteristics. But due to the increased filter capacitance, the current and voltage amplitude increase slightly, and the impact of the transient current shock is still higher than the grid current transient index, so this method cannot fully meet the needs.

Fig. 5 shows the grid voltage and current waveform of the system adopting the current rate control method when CPL is suddenly put into the grid. Fig. 5 (1) shows the condition of $\left|k_{2}\right|>1$, and the oscillation of the system time is shorter, but because $\left|k_{2}\right|>1$, the damping coefficient decreases, which causes overshoot volume and dynamic transient impact amplitude increase during the adjusting process. Fig. 5 (2) and Fig. 5 (3) Fig. 5 (1) show the condition of $\left|k_{2}\right|<1$. Along with $k_{2}$ decreases gradually, the transient process of power grid gradually gets improved. When $k_{2}$ is 
small enough, grid transient impact and shock can be basically eliminated. However, with the decrease of $k_{2}$, the response time increases, so the system rapidity is adversely affected. To solve the problem, Fig.5 (4) adopts an improved rate control method, in which a limiting link is added to the current rate of change loop. In this method, the value of $k_{2}$ could be increased appropriately, and current rate of change can be limited by the limiting link. Comparing Fig. 5 (2) and (4), when $k_{2}$ is the same, the limiting link can control the current rate of change in a reasonable scope, and thus increase the equivalent dynamic resistance and eliminate the shock and impact in dynamic process. Comparing Fig. 5 (3) and (4), $k_{2}$ can increase appropriately and the dynamic response gets faster on the premise of smooth transition when the current rate of change control method is used. This is because the current change is limited by the limiting link to solve the problem of grid turbulence in the early dynamic response process, and what's more, a larger $k_{2}$ helps the current keep a fast regulating speed in the late dynamic response process to ensure the rapidity of the grid.

Through the above analysis and simulation results, the current rate of change control method can satisfy the requirements of the grid much better than changing the parameters of EMI filter.

\section{Conclusions}

A kind of new control method is proposed in this paper to improve aviation power grid stability. Current rate of change controlling link with limiting link is adopted in the closed-loop control system of CPL. By the control of current rate of change, equivalent dynamic load resistance of CPL is adjusted in a proper scope so as to eliminate the transient impact and shock of grid voltage and current in the event of a disturbance and improve the dynamic characteristics of power grid.

\section{References}

[1] Wildrick C. M., Lee F. C., et al. A method of defining the load impedance specification for a stable distributed power system[J]. IEEE Transactions on Power Electronics, 1995, V10(3): 280-285

[2] Liu, X., Andrew, J., Andrew, M.: 'Negative input-resistance compensator for a constant power load', IEEE Tran. Ind. Electron., 2007, 54, (6), pp. 3188-3196

[3] Du W J,Zhang J M,Zhang Y,et al.Stability criterion for cascaded system with constant power load[J] . IEEE Trans.on Power Electronics,2013,28(4):1843-1851.

[4] Belkhayat M,Cooley R,Abed E H.Stability and dynamics of power systems with regulated converters [J].Circuits and Systems, 1995,1(28):143-145. 